\title{
ПРИМЕНЕНИЕ ИНТЕЛЛЕКТУАЛЬНЫХ СИСТЕМ ДЛЯ РЕШЕНИЯ ЗАДАЧ В ОБЛАСТИ ГЕОПРОСТРАНСТВЕННЫХ ТЕХНОЛОГИЙ И ДИСТАНЦИОННОГО ЗОНДИРОВАНИЯ
}

\section{Татьяна Юрьевна Бугакова}

Сибирский государственный университет геосистем и технологий, 630108, Россия, г. Новосибирск, ул. Плахотного, 10, кандидат технических наук, доцент кафедры прикладной информатики и информационных систем, тел. (383)343-18-53, e-mail: kaf.pi@ssga.ru

\section{Артем Андреевич Шарапов}

Сибирский государственный университет геосистем и технологий, 630108, Россия, г. Новосибирск, ул. Плахотного, 10, ассистент кафедры прикладной информатики и информационных систем, тел. (953)785-54-99, e-mail: sharapov_artem@mail.ru

Настоящее время характеризуется кардинальными изменениями в технологическом развитии общества во всех сферах деятельности человека. Интеллектуальные системы стремительно развиваются и заменяют ручную обработку данных и существующие автоматизированные системы. Развитие интеллектуальных систем касается геодезии, картографии, кадастра, геоинформатики и др. Интеллектуальные системы постепенно внедряются в кадастровую сферу деятельности, в то время как в геодезии и картографии в основном применяются классические методы обработки и получения данных. В статье рассмотрен пример обучения нейронной сети для распознавания объектов на растровом изображении.

Ключевые слова: интеллектуальные системы, искусственный интеллект, геоинформационные системы, нейронные сети, обработка данных, дистанционное зондирование, интеллектуальный анализ

\section{APPLICATION OF AI SYSTEMS FOR SOLVING PROBLEMS IN THE FIELD OF GEOSPATIAL TECHNOLOGIES AND REMOTE SENSING}

\section{Tatiana Yu. Bugakova}

Siberian State University of Geosystems and Technologies, 10, Plakhotnogo St., Novosibirsk, 630108, Russia, Ph. D., Head of the Department of Applied Informatics and Information Systems, phone: (383)343-18-53, e-mail: kaf.pi@ssga.ru

\section{Artem A. Sharapov}

Siberian State University of Geosystems and Technologies, 10, Plakhotnogo St., Novosibirsk, 630108, Russia, Assistant of the Department of Applied Informatics and Information Systems, phone: (953)785-54-99, e-mail: sharapov_artem@mail.ru

The present time is characterized by cardinal changes in the technological development of society in all spheres of human activity. Intelligent systems are rapidly evolving and replacing manual data processing and existing automated systems. The development of intelligent systems also concerns geodesy, cartography, cadastre, geoinformatics, etc. AI systems are gradually being introduced into the cadastral sphere of activity, while in geodesy and cartography, classical methods of obtaining and processing data are mainly used. The article discusses an example of training a neural network for recognizing objects in a raster image.

Keywords: AI systems, artificial intelligence, geographic information systems, neural networks, data processing, remote sensing, intelligent analysis 
Сейчас человечество стоит на рубеже кардинальных изменений методологических и технологических решений во всех сферах деятельности. Связано это с тем, что в настоящее время технологии вышли на новый уровень развития. Управление сложными технологическими процессами определило необходимость минимизации человеческого фактора путем активного внедрения интеллектуальных систем во все технологические и управленческие процессы. Одной из приоритетных задач федерального проекта «Цифровые технологии» национальной программы «Цифровая экономика Российской Федерации» является внедрение искусственного интеллекта во все сферы человеческой деятельности [1].

Развитие интеллектуальных систем касается геодезии, картографии, кадастра, геоинформатики и др. Эволюционное развитие этих сфер деятельности, как и других, определяется переходом от «ручных» измерений и обработки информации к автоматизированным системам. В настоящее время технологический прогресс находится на переломном уровне - переходе от автоматизированных систем к интеллектуальным, основой которых является искусственный интеллект [2].

Под искусственным интеллектом сегодня принято понимать всю совокупность методов, с помощью которых создаются интеллектуальные системы: машинное обучение, нейронные сети, технологии распознавания естественного языка, обработка голоса, компьютерное зрение и т. д.

В настоящее время в кадастре и дистанционном зондировании все чаще возникают задачи по интеллектуальной обработке и анализу изображений, в том числе снимков, используемых в геоинформационных системах. Одним из интеллекутальных методов для решения такого рода задач является машинное обучение, как одно из средств искусственного интеллекта. В программных продуктах для обработки геоинформационных данных начинают появляться инструменты для интеллектуального анализа данных.

Например, в программном продукте ArcGIS разработаны модули, основанные на современных алгоритмах для принятия решений и анализа данных. Модули включают в себя инструментарий, позволяющий реализовать модели машинного обучения путем включения пространственных данных и алгоритмов, выполнять классификацию данных дистанционного зондирования. B ArcGIS на текущий момент доступны следующие архитектуры нейронных сетей:

- SSD (Single Shot Detector) - нейронная сеть, позволяющая выполнять поиск и классификацию объектов на изображении (рис. 1);

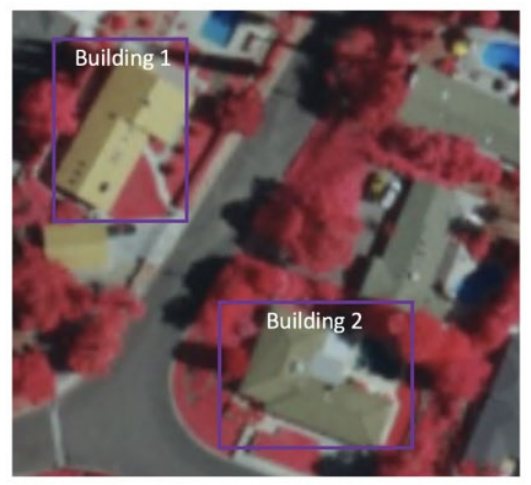

Рис. 1. Нахождение объекта на снимке нейронной сетью SSD 
- U-net - позволяет выполнить классификацию каждого пикселя растрового снимка (рис. 2);
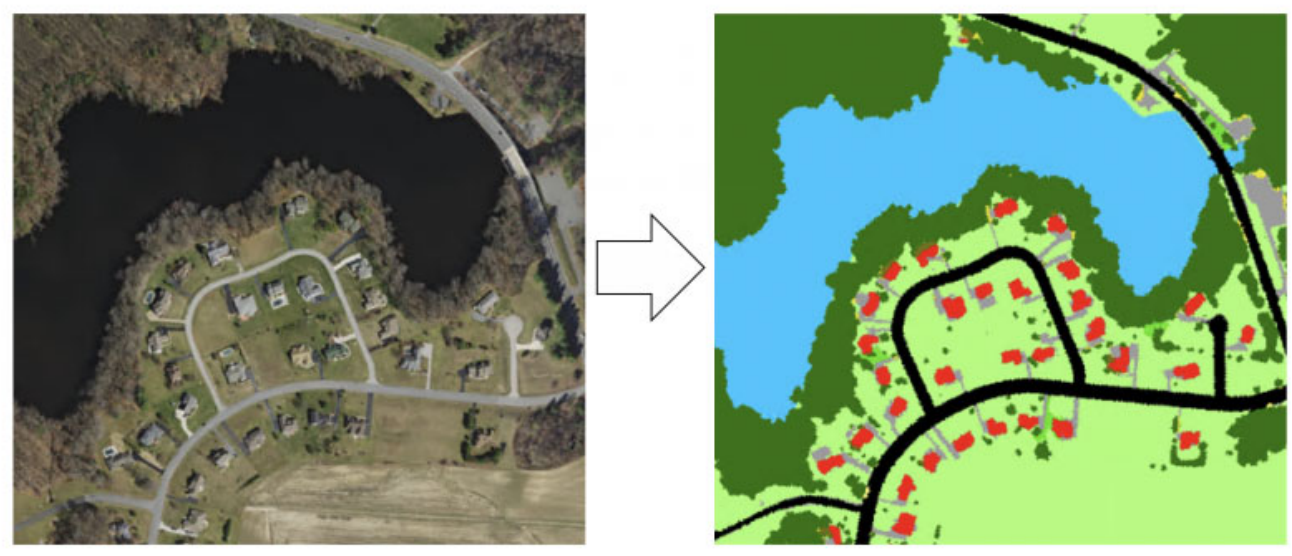

Рис. 2. Результат работы нейронной сети U-net

- MaskRCNN - выполняет поиск объектов на фоне и дополнительно строит для них маски (рис. 3);

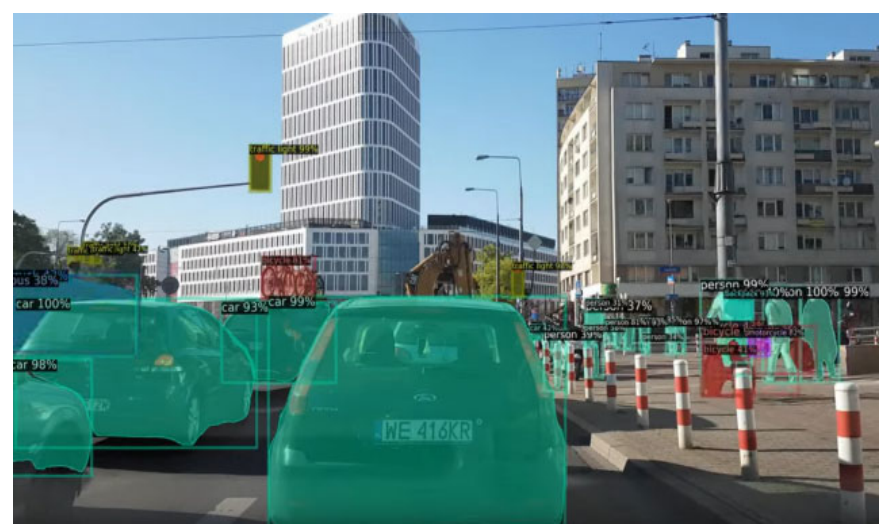

Рис. 3. Сегментация изображения

- Named Entity Extraction - нейронная сеть, функционирующая на базе библиотеки spaCy, которая предназначена для извлечения информации из неструктурированного текста [7].

В качестве входных данных для нейронной сети используются пространственные данные, в том числе пространственные данные ГИС. Нейросетевые алгоритмы динамически модифицируют характеристики существующих объектов, отображенных в ГИС, и могут создавать новые объекты и даже новые слои карт.

Однако, подобные программные продукты, включающие в свой состав возможность применения нейронных сетей, довольно дороги. Далеко не каждая организация, а тем более начинающий исследователь может позволить себе купить лицензию. 
Еще одним ограничивающим фактором в использовании данного вида программного обеспечения является переход к Российскому программному обеспечению по программе импортозамещения с целью обеспечения технологической независимости критической информационной инфраструктуры.

Таким образом, остро стоит вопрос о разработке собственных программных продуктов и алгоритмов, основанных на применении нейронных сетей и машинного обучения для задач кадастра, геодезии и картографии [4].

На данный момент авторами ведутся исследования технологий искусственного интеллекта и выполнен эксперимент по разработке нейронной сети на примере распознавания зданий и сооружений на растровом изображении.

Основными инструментами для обучения нейросети являются языки программирования Python и TensorFlow - открытая программная библиотека для машинного обучения, разработанная компанией Google для решения задач построения и тренировки нейронной сети с целью автоматического нахождения и классификации образов, достигая качества человеческого восприятия [8].

Изначально необходимо выполнить обучение нейронной сети на основе подготовленного набора данных, для выполнения семантической сегментации растрового изображения. Семантическая сегментация изображений - это разделение изображения на отдельные группы пикселей, области, соответствующие одному объекту с одновременным определением типа объекта в каждой области. Именно при помощи сегментации изображения нейронная сеть определяет принадлежность того или иного пикселя изображения к искомой группе объектов, в данном случае зданиям и сооружениям [5].

В качестве сети для обучения была взята Mask R-CNN, данная сеть подходит для обнаружения объектов. Подготовка dataset выполнялась в VGG Image Annotator - сервис, позволяющий выполнить разметку изображений для дальнейшего обучения нейронной сети (рис. 4).

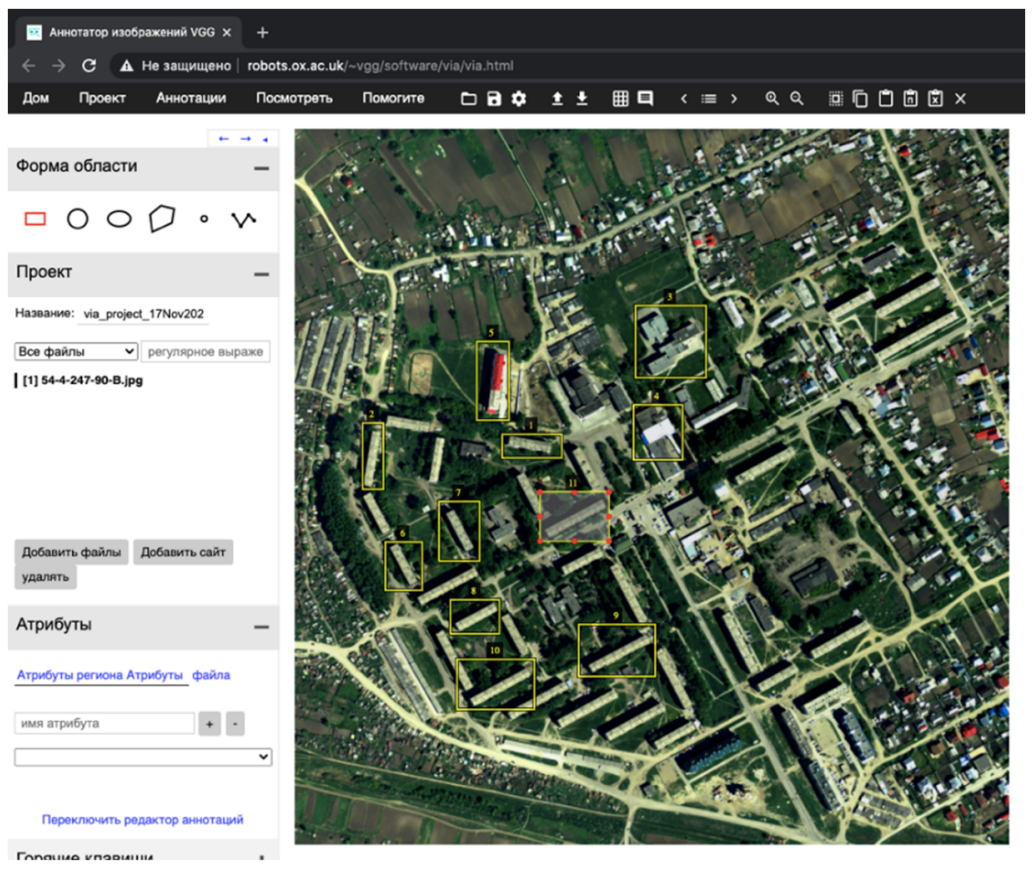

Рис. 4. Процесс разметки изображений 
Далее на подготовленном наборе данных была обучена сеть. Огромное значение при обучении играет качество подготовки данных, их полнота и условия, в которых они были получены. Например, если фотоснимок был получен в зимнее время года, то нейронная сеть не определит или определит здания на снимке, но с большой погрешностью. Для устранения этой проблемы требуется дополнительное обучение на основе снимков, которые были получены в зимнее время года. В целом, точность построения модели напрямую зависит от качества обучения нейронной сети. Чем больше исходных данных, тем выше точность определения объектов на изображениях нейронной сетью.

Результат работы нейронной сети представлен на рис. 5.

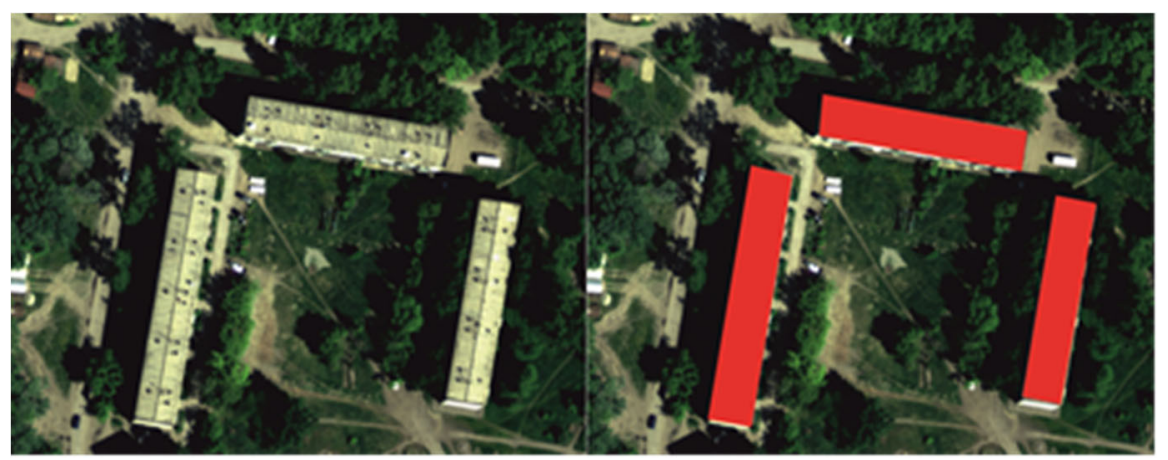

Рис. 5. Результат работы обученной нейронной сети

В отличие от общеизвестных классических методов, которые основаны на математических вычислениях, методы, работающие средствами нейронных сетей способны адаптироваться в процессе обучения и построить математическое обоснование цифровой модели местности.

Технологии искусственного интеллекта отлично подходят для решения разного рода задач. В кадастровой деятельности применение нейронных сетей позволит оптимизировать время проведения оценки объектов недвижимости, своевременно находить нарушения градостроительного регламента, путем обнаружения незаконных построек или построенных с нарушениями. Перспективой применения нейронных сетей в геодезическом производстве является их использование для оперативной обработки больших массивов данных лазерного сканирования. В настоящее время обработка большого объема данных лазерного сканирования - трудоемкая операция, занимающая значительное количество времени. Нейронные сети позволят оперативно обработать большие массивы данных, классифицировать группы точек по определенным категориям объектов, что значительно упростит дальнейшую обработку и анализ данных.

Перспективой применения нейронных сетей в картографии является интеллектуальная оцифровка фрагмента растрового изображения (например, лесного массива, водоемов, горной местности и других), что, по сравнению с используемыми методами, позволяет значительно снизить шумы и выполнять интеллектуальную оцифровку снимка низкого качества. 


\section{БИБЛИОГРАФИЧЕСКИЙ СПИСОК}

1. А. Н. Васильев, Д.А.Тархов. Нейросетевое моделирование. Принципы. Алгоритмы. Приложения. СПб.: Изд-во Политехн. Ун-та, 2009. ISBN 978-5-7422-2272-9.

2. С. А. Шумский. Машинный интеллект. Очерки по теории машинного обучения и искусственного интеллекта. М., РИОР, 2019. DOI: 10.29039/02011-1.

3. Д. А. Тархов. Нейросетевые модели и алгоритмы. Справочник. М., Радиотехника, 2014.

4. Дьяченко Н.В. Опыт разработки информационно-аналитических систем поддержки принятия управленческих решений - http: // www. nocnit. ru/2st/materials/Diachenko. Html.

5. Капралов Е.Г., Кошкарев А.В., Тикунов В.С. и др. Основы геоинформатики. Уч. пособие. - М.: Изд. центр «Академия», 2004. -480 с.

6. Алешин Л.И. Информационные технологии. - Учебное пособие. М: Московская финансово-промышленная академия, 2008.

7. Что такое Deep Learning? - https://blogs.esri-cis.ru/2020/03/19/arcgis-\%D0\%B8-deep-learning/.

8. Спутниковые снимки - https://yandex.ru/support/nmaps/sputnik.html.

(C) T. Ю. Бугакова, А. А. Шарапов, 2021 